Simons, M., Koopman, H. \& Osinga, M. (2013). Would you fly with a pilot on insulin?. Lancet

Diabetes \& Endocrinology, Early Online Publication, 9 December 2013

http://dx.doi.org/10.1016/S2213-8587(13)70197-9

\title{
Comment
}

\section{Would you fly with a pilot on insulin?}

Insulin treatment affects the careers of diabetic professionals doing safety-sensitive jobs, such as airline pilots. In most cases, insulin-treated individuals are automatically banned from safety-sensitive jobs because the risk of hypoglycaemia is regarded as incompatible with safety. Recent developments, such as diabetes selfmanagement education, self-monitoring of blood glucose, continuous glucose monitoring, and shortacting and longacting insulin analogues enable better achievement of individual glycaemic targets and prevention of hypoglycaemia than did management options available previously. ${ }^{1}$ These developments justify reconsideration of the safety risks of airline pilots on insulin.

Some national authorities (USA, UK, Canada, and Australia) and the International Civil Aviation Organisation have regulatory provisions for private pilots, but worldwide, all but two civil aviation authorities disqualify insulin-treated commercial pilots. On a case-by-case basis, the Canadian civil aviation authority considers commercial pilots requiring insulin to fly, and several Canadian insulin- treated pilots are flying under intensive aeromedical control. Because European rules on this issue have not been put in place by the European Aviation Safety Agency (EASA), the UK civil aviation authority has tried to speed up rule-making processes by issuing a protocol that could allow commercial pilots requiring insulin to fly under strict conditions. ${ }^{2}$ This protocol, effective since 2013 , is only used by the UK civil aviation authority and not by other European countries, thereby creating a discrepancy: pilots deemed safe to fly in the UK are regarded as unsafe in other countries. As a consequence, several non-British insulin-treated pilots apply to the UK civil aviation authority for their medical certification. We reason that the protocol of the UK civil aviation authority can safely be introduced by EASA and other authorities, and that the $\mathrm{HbA}_{1 \mathrm{c}}$ of $7.5-8.5 \%$ (58-69 $\left.\mathrm{mmol} / \mathrm{mol}\right)$, used in the UK protocol, could safely be lowered to less than $7 \%(53 \mathrm{mmol} / \mathrm{mol})$, as recommended by the American Diabetes Association (ADA) and the European Association for the Study of Diabetes (EASD) to optimally reduce the risk of microvascular and macrovascular complications. ${ }^{3}$

Intensive glucose-lowering treatment can increase the risk of hypoglycaemia. ${ }^{3}$ When blood glucose reaches less than roughly $3.1 \mathrm{mmol} / \mathrm{L}$, symptoms of neuroglycopaenia and cognitive impairment develop, which are believed to interfere with flying abilities and alertness. ${ }^{4}$ However, because insulin-treated professionals have always been automatically banned from armed and emergency services, train driving, professional diving, and off-shore oil rig work, little or no data for safety risks of their insulin treatment exist. Similarly, no data are available for the effects of insulin treatment on flight safety. Based on data for crash risks of truck drivers and the effects of experimental hypoglycaemia on driving capacities, the second European working group on diabetes and driving concluded that the accident risk for commercial truck drivers is acceptable when cases of hypoglycaemic unawareness or severe hypoglycaemia are excluded and on-the-job self-monitoring of blood glucose guarantees blood glucose between $5.5 \mathrm{mmol} / \mathrm{L}$ and $22 \mathrm{mmol} / \mathrm{L}$. ${ }^{5}$ Based on results from studies of the effects of experimental hypoglycaemia on complex tasks, ${ }^{4}$ we propose a blood glucose of $5 \mathrm{mmol} / \mathrm{L}$ to be the lower limit for safe functioning of pilots.

To be eligible for possible aeromedical certification, pilots should show the following evidence of stable glycaemic control: $\mathrm{HbA}_{1 \mathrm{C}}$ between 6.5 and $8.0 \%$ (42-64 mmol/mol); blood glucose analysis over 3 months before application ( $\leq 5 \%$ of readings $<4.0 \mathrm{mmol} / \mathrm{L}$ and $80 \%$ of readings between 5 and $15 \mathrm{mmol} / \mathrm{L}$ ); and no recurrent ( $\geq$ two) episodes of severe hypoglycaemia in the past 5 years and none in the preceding 1 year. Notably, the ADA/EASD recommendations suggest that a $\mathrm{HbA}_{1 \mathrm{c}}$ concentration of between 6 and $7 \%(42-53$ 
$\mathrm{mmol} / \mathrm{mol}$ ) enables pilots to have safe in-flight blood glucose, because these $\mathrm{HbA}_{1 \mathrm{c}}$ values are associated with average estimated glucose concentrations of between $7.0 \mathrm{mmol} / \mathrm{L}$ and $8.6 \mathrm{mmol} / \mathrm{L} .{ }^{1,6}$

During mandatory simulator checks, pilots in two-pilot airline operations have to prove they can safely operate the aircraft in case the other pilot is incapacitated. When airline pilots do not fully meet the medical certificate requirements but are considered to be within the accepted risk of incapacitation, they can be allowed to fly "as or with a qualified co-pilot" (EASA: Operational Multicrew Limitation). Such a restriction should also apply to insulin-treated pilots; co-piloting also provide sufficient opportunities to measure and manage pre-flight and in-flight blood glucose. Management of diabetes in airline pilots also needs to anticipate adjustments due to time zone crossings, night duties, irregular work, and delays. Professional coaching and self-monitoring of blood glucose in combination with longacting insulin analogues to control glycaemic concentrations between meals and rapid-acting analogues to control postprandial hyperglycaemia, seem not only to improve glycaemic control and prevention of hypoglycaemia, but will also enable coping with above-mentioned specific operational problems. ${ }^{1,7-9}$

Long duration of diabetes is associated with increased risk of reduced hypoglycaemia awareness thereby increasing the risk of severe hypoglycaemia, although these risks might be mitigated by long-term stable glycaemic control. In the context of safety, we recommend the consideration of regular checks of pilots to identify reduced hypoglycaemia awareness with hyperinsulinaemic-hypoglycaemic clamp tests. These tests can provide subjective (symptoms, cognitive functioning) and objective (counterregulatory hormones) evidence for reduced hypoglycaemic awareness. ${ }^{10}$

In accordance with the UK civil aviation authority protocol, it is recommended to require pilots to regularly measure and manage their blood glucose before and during the flight and to keep their pre-flight and in-flight blood glucose between 5 and $15 \mathrm{mmol} / \mathrm{L}^{2}$ The memory of the test meter, preferably a (real time) continuous glucose monitoring system, should be periodically reviewed to ensure protocol compliance. ${ }^{2}$ In case flightoperational demands have priority over measuring blood glucose, the pilot should ingest a glucose snack and measure the blood glucose 30 min later. ${ }^{2}$

We will confidently fly with an insulin-treated pilot who has a stable glycaemic control, has no substantial disease complications, and is compliant with self measurement and management of pre-flight and in-flight blood glucose. Civil aviation authorities should continue to aim for consistency in guidelines between international authorities and consider individual cases of insulin-treated pilots for certification. Data for all pilots flying with insulin should be systematically collected to assess the usefulness and safety of the recommendations.

\section{Ries Simons, Henk Koopman, Marja Osinga}

European Society of Aerospace Medicine, TNO Human Factors, 3769 DH Soesterberg, Netherlands (RS); Aviation Medical Centre, 6199 AD Maastricht-Airport, Netherlands (HK); and Aeromedical Expert Centre, 1438 AN Oude Meer, Netherlands (MO)

ries.simons@tno.nl

We declare that we have no conflicts of interest. All authors did the literature study and contributed to writing of the report. RS had discussions with the authorities.

1. American Diabetes Association. Standards of medical care in diabetes-2011. Diabetes Care 2011; 34 (suppl 1): 11-61.

2. UK Civil Aviation Authority, Medical Department. Diabetes Certification Guidance 03/ 2013 v2. 0. http:// www. caa.co.uk/ docs/ 49/20130226DiabetesCertificationGuidanceFeb13. pdf (accessed 19 Nov, 2013).

3. Nathan DM, Buse JB, Davidson MB, et al. American Diabetes Association, European Association for Study of Diabetes. Medical management of hyperglycemia in type 2 diabetes: a consensus algorithm for the initiation and adjustment of 
therapy: a consensus statement of the American Diabetes Association and the European Association for the Study of Diabetes. Diabetes Care 2009; 32: 193-203.

4. Warren RE, Frier BM. Hypoglycaemia and cognitive function. Diabetes Obes Metab 2005; 7: 493-503.

5. Berne C, Sheppard D, Boyd B, Albrecht M, et al. Diabetes and driving in Europe. A report of the Second European Working Group on Diabetes and Driving 2006, Brussels. J uly 2006.

http:// ec. europa. eu/transport/ road safety/ behavior/doc/ diabetes and driving in europe final 1 en. pdf (accessed Nov 19, 2013).

6. Nathan DM, Kuenen J, Borg R, Zheng H, Schoenfeld D, Heine RJ, for the Alc-Derived Average Glucose Study Group. Translating the A1C assay into estimated average glucose values. Diabetes Care 2008; 31: 1473-78.

7. Rosenstock J, Dailey G, Massi-Benedetti M, Fritsche A, Lin Z, Salzman A. Reduced hypoglycemia risk with insulin glargine: a meta-analysis comparing insulin glargine with human NPH insulin in type 2 diabetes. Diabetes Care 2005; 28: 950-55.

8. Heller S, Buse J, Fisher M, et al, for the BEGIN Basal-Bolus Type 1 Trial Investigators. Insulin degludec, an ultra-longacting basal insulin, versus insulin glargine in basal-bolus treatment with mealtime insulin aspart in type 1 diabetes (BEGIN BasalBolus Type 1): a phase 3, randomised, open-label, treat-to-target non-inferiority trial. Lancet 2012; 379: 1489-97.

9. Garber AJ , King AB, Del Prato S, et al. NN1250-3582 (BEGIN BB T2D) Trial Investigators. Insulin degludec, an ultralongacting basal insulin, versus insulin glargine in basal-bolus treatment with mealtime insulin aspart in type 2 diabetes (BEGIN Basal-Bolus Type 2): a phase 3, randomised, open-label, treat-to-target non-inferiority trial. Lancet 2012; 379: 1498507.

10. Leelarathna L, Little SA, Walkinshaw E, Tan HK, et al. Restoration of Self-awareness of Hypoglycemia in Adults With Long-standing Type 1 Diabetes: Hyperinsulinemic-hypoglycemic clamp substudy results from the HypoCOMPaSS trial. Diabetes Care 2013; published online Oct 15. DOI:10.2337/ dc13-1004. 